\title{
Attachment-retained Unilateral Distal Extension (Kennedy's Class II Modification I) Cast Partial Denture
}

\author{
Ashish R Jain, Jacob Mathew Philip, Padma Ariga
}

\begin{abstract}
Attachment-retained removable partial denture (RPD) is not an outdated treatment modality. It is even more contemporary in today's appearance-oriented society than when it was first introduced. There is significant number of patients who could benefit from this treatment option, both short and long term. However, lack of proper knowledge, overwhelming number of attachments available in the market, multiple adjustments and repairs are making dentists reluctant to offer and provide attachment-retained RPD services. The purpose of this article is to provide an overview and a simplified approach to this treatment modality by way of a case report.
\end{abstract}

Keywords: Attachment, Removable partial denture.

How to cite this article: J ain AR, Philip J M, Ariga $P$. Attachment-retained Unilateral Distal Extension (Kennedy's Class II Modification I) Cast Partial Denture. Int J Prosthodont Restor Dent 2012;2(3):101-107.

Source of support $\mathrm{Nil}$

Conflict of interest: None

\section{INTRODUCTION}

The clinical use of a unilateral removable partial denture (RPD) is limited because of its poor stability and retention. A regular problem faced by the partially edentulous patient is the nuance of adapting to a removable prosthesis. A unilateral prosthesis is always less stable, because it lacks the effect of cross arch stabilization. Rehabilitation of K ennedy's Classes I and II partially edentulous patients can be challenging because a natural tooth retained fixed prostheses cannot be fabricated. I mplant-retained restoration is an option but this is sometimes not possible due to insufficient amount of bone or economic reasons. In these cases, acrylic or cast partial denture have been largely preferred, but with barely satisfactory esthetical results. Precision attachment has long been considered the highest form of partial denture therapy. A $n$ alternative reconstructive option that does not involve complex procedures for the patient is combination prosthesis with fixed and removal partial denture connected with attachments. This prosthetic option, in addition to the esthetics and functional advantage of a fixed denture, gives a decreased compression of the edentulous ridge and enhanced mastication and phonetics. The few retrospective studies available show a survival rate of $83.3 \%$ for 5 years, of $67.3 \%$ up to 15 years and of $50 \%$ when extrapolated to 20 years ${ }^{1,2}$
A ttachment retained RPD is a viable treatment alternative through which a significant number of patients could be benefited. In this particular case, an attachmentretained RPD was chosen as a treatment modality. This article provides an overview and a simplified approach to this treatment option.

\section{CLASSIFICATION OF ATTACHMENTS}

An attachment is a connector consisting of two or more parts. One part is connected to a root, tooth or implant and the other part to prosthesis. Precision attachments can be classified in to four main groups ${ }^{3}$ :

1. Intracoronal attachments are mainly used in connecting units of fixed partial prostheses, retaining restorations with distal extension or bounded removable prostheses.

2. Extracoronal attachments- this type of attachment provides stability and retention for removable distal extension prostheses.

3. Stud attachments- usually in the form of ball and socket, this attachment serves primarily for overdenture stabilization and retention of the prosthesis. Swiss logic, ZA A G, Zest anchor is example of stud attachments. One of the advantages of stud is that they promote better oral hygiene and crown-root ratio is improved with low profile stud attachments.

4. Bar attachments-originally used for splinting groups of teeth, currently used for overdenture retention and stabilization.

\section{Functional Classifications}

- Class 1A - solid, rigid, nonresilient

- Class 1B-solid, rigid-lockable

- Class 2-vertical resilient

- Class 3- hinge resilient

- Class 4-vertical and hinge resilient

- Class 5-rotational and vertical resilient

- Class 6- universal, omniplanar.

Indications for Attachment-retained Partial Denture

- Esthetics

- Redistribution of forces

- Minimize trauma to soft tissue 
- Control of loading and rotational forces

- Nonparallel abutments-Segmenting

- Future salvage efforts-Segmenting

- Retention.

\section{Contraindications for Attachment-retained Partial Denture}

Short clinical crowns prove to be the foremost contraindication to the use of attachments in the construction of RPDs. The tooth must have adequate crown height to house the attachment components and effectively offset the leverage forces exerted on the crown. In addition, adequate height must be present for the corresponding attachment components to be housed within the RPD framework or supportive acrylic resin while allowing an optimal artificial tooth placement. ${ }^{1-6}$

\section{What are the Advantages of Precision Attachment Cases?}

Precision attachment partial dentures are the very best restorations where fixed restorations are contraindicated. Experience with more than 1,000 cases during the last 50 years has uncovered several key advantages of this type of restoration:

1. Cosmetic appearance.

2. M aintainable periodontal health.

3. L ongevity of abutment teeth.

4. Patient comfort.

5. Questionable teeth can be saved in a way that does not affect case.

6. Longevity if they are lost in the future.

7. Natural tooth and/or implant abutments can be used.

8. Precision attachment partial dentures can be adapted to compensate for future changes in the mouth.

\section{How to choose an Attachment?}

When selecting an attachment, dentist wishes to use the best attachment in specific cases. There is probably no such thing as 'best attachment' but there may be several attachments that will work equally well. So, one should not select an attachment by name rather by understanding basic principles, which never change. Selection principles are based on the following:
a. Crown root ratio desired
b. Type of copings
c. Vertical space available
d. Number of teeth support
e. A mount of bone support
f. Location of abutments
g. Location of strongest abutment
h. Type of opposing dentition
i. Maintenance problems
j. Cost

\section{How does a Precision Partial Stay in Place?}

Even though there are no locking mechanisms, a good precision attachment partial denture will not dislodge during normal function. The reason the partial denture does not continually fall out is because it is surveyed in two directions so that the path of insertion is different from the pull of the muscles and the action of the tongue and gravity. A lthough the partial cannot be dislodged during function, it can move in a vertical direction slightly to release the forces instead of passing along these forces to the abutment teeth. The result is physiologic stimulation of the abutment teeth and the edentulous ridges. Clinical experience has shown that this physiologic stimulation results in increased longevity of the abutment teeth, ${ }^{4}$ even when a few teeth are required to carry the load of an entire arch. The stimulation of the edentulous ridge also prevents the bone resorption that typically reduces tissue support for the partial denture. The tissue under a well-fitting precision attachment partial is typically healthy and firm. There is surprisingly little wear of attachments that are used in this manner, even after many years of function! While the prosthesis may require relining or alteration of the occlusion to compensate for changes, it is rarely necessary to adjust or replace the male attachments on the partial. ${ }^{4}$ The partial is never kept in a glass overnight. It is worn 24 hours a day to prevent collapse of the musculature and the occlusion and only removed for hygiene.

\section{What are the Requirements for Precision Attachment Partial Dentures?}

The success of precision attachment partial dentures depends on creating an ideal architecture for the mouth. A ll the teeth that support bridgework are prepared with full shoulder preparations (flat ledges) in three-dimensions. In other words, the prepared teeth relate to each other, the gingiva (gums) and the underlying bone. A ny defects must be corrected surgically in order to create health and the best architectural foundation to support dentistry. The dentistry must conform to sound principles of biomechanics that minimize forces on this foundation. These principles include narrow occlusal (biting surface) diameters, adequate room for hygiene, proper length and anatomy, solid and passive fit and adequate occlusion (bite) at the correct jaw relationship. The importance of accurate impressions and models to the overall success of a precision attachment case cannot be overemphasized. Precision attachments must be used in a precise manner in order to maintain a high 
percentage of longevity. Great care must be taken to ensure precision at every step; and steps cannot be skipped or the final result will be compromised. The entire chain can only be as strong as the weakest link. Precision attachment cases must fit with precision - the abutments must be stable and the frameworks must fit properly without rock.

\section{Abutment Tooth Preparation}

There are five main steps involved in preparation of abutment teeth to receive full coverage castings with precision attachment retained systems. All these procedures are employed when any other intracoronal retainer system is employed. ${ }^{7-9}$

1. Surveying the diagnostic cast to ensure proper placement of required precision attachment for esthetics, periodontal health, restoration of biologic tooth contours and an optimal path of insertion of the RPD.

2. Prepare guide planes to avoid an overcontoured restoration on these surfaces of subsequent restoration.

3. Prepare a box or deep recess to house the precision attachment rest seat so as to avoid an overbuilt casting.

4. Prepare the entire abutment tooth using a full shoulder with a bevel type of preparation. When preparing a tooth for any intracoronal attachment the dentist must deepen the shoulder from the midpoint of the buccal surface to the midpoint on the lingual surface, on the near proximal zone (the area adjacent to edentulous area, whereas the far proximal zone is the area away from the edentulous area. This area is needed for technician to maneuver the attachment on the surveyor for proper parallelism and placement of the precision attachment and lingual sleeve without over contouring the restoration in relation to the other precision attachments employed in that particular situation.

5. Add grooves/boxes to increase the resistance to dislodgment on short crowned abutment preparations or on those ravaged by caries or large previous restoration.

\section{CLINICAL REPORT}

A 45-year-old female reported to our outpatient clinic (Saveetha Dental College And Hospitals, Saveetha University, Chennai, India) with chief complaint of missing teeth in right and left lower posterior region of the jaw and inability to eat food. She was wearing a distal extension RPD (missing 36,37) and the presence of mandibular extracoronal clasp retainers was negatively affecting the esthetics. The patient's medical history was evaluated and was found to be noncontributory. The missing teeth were $16,26,27,36,37,46,47$ (Figs $1 A$ and B). Several treatment options were offered to the patient: A RPD, an implant-
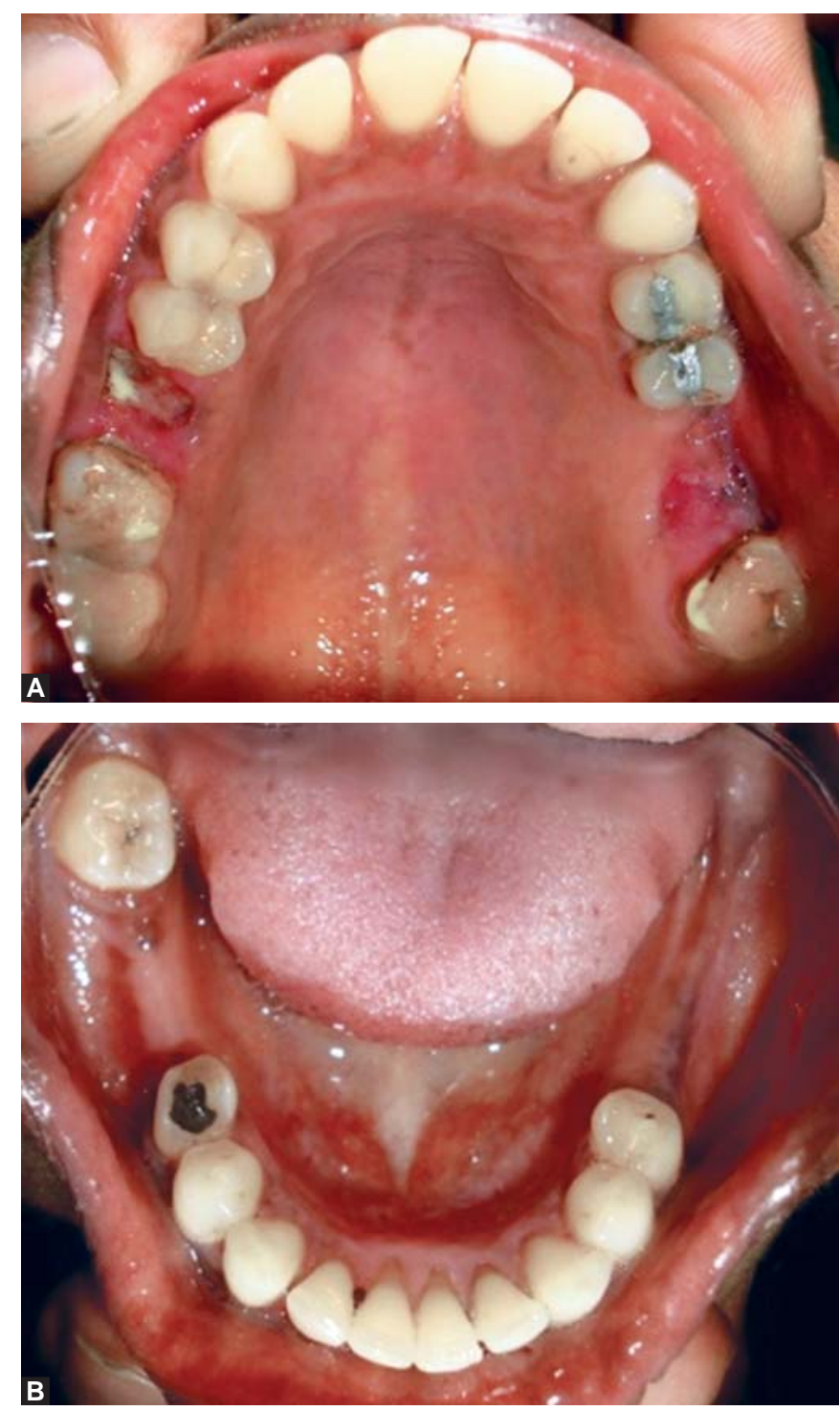

Figs 1A and B: P reoperative photograph after immediate extraction

supported prosthesis, combination of a fixed prosthesis with RPD and combination denture with fixed and removal prosthesis with attachments. A fter reviewing the options, the patient accepted the latter treatment option. The patient rejected the options of implants because of the need for additional surgery and the unacceptable duration of treatment phase.

\section{TECHNIQUE}

- Diagnostic impressions were made and mounted on semiadjustable articulator using a face bow. Following which diagnostic wax-up was done on the mounted casts.

- A putty matrix (Express STD Putty, 3M ESPE, St Paul, $M N, U S A$ ) was made over the completed diagnostic wax-up for evaluation of the existing space for the extracoronal resilient attachment.

- The attachment system was selected on the basis of available space (OT CAP, R hein 83 Inc. USA). 
- Tooth preparation was done on $15,17,25,28,34,35$ and 45 to receive PFM crowns. Rest seat were prepared in 48 mesially and rest seat in 45 was milled in PFM crown distally. Impression was made and poured in die stone. Following which crowns have been waxed to full contour and milled in wax for maximum guiding plane surface. The patrices was added to the axial surfaces of the abutment using a dental surveyor, lingual to the center of proximal contour. This ensures that the bulk of matrice does not interfere with esthetic of buccal cusp of replacing denture tooth.

- Metal try-in of the coping was done to evaluate the fit of the casting. Ceramic layering was done on the metal frame work tried (Figs $2 A$ and $B$ ).

- The fabricated metal ceramic crowns were provisionally cemented with the patrix attached to the casting and picked up using putty impression for fabricating the removal partial denture (Figs $3 \mathrm{~A}$ to $\mathrm{C}$ ). M axillary FPD was permanently cemented with glass ionomer cement (Fig. 4).
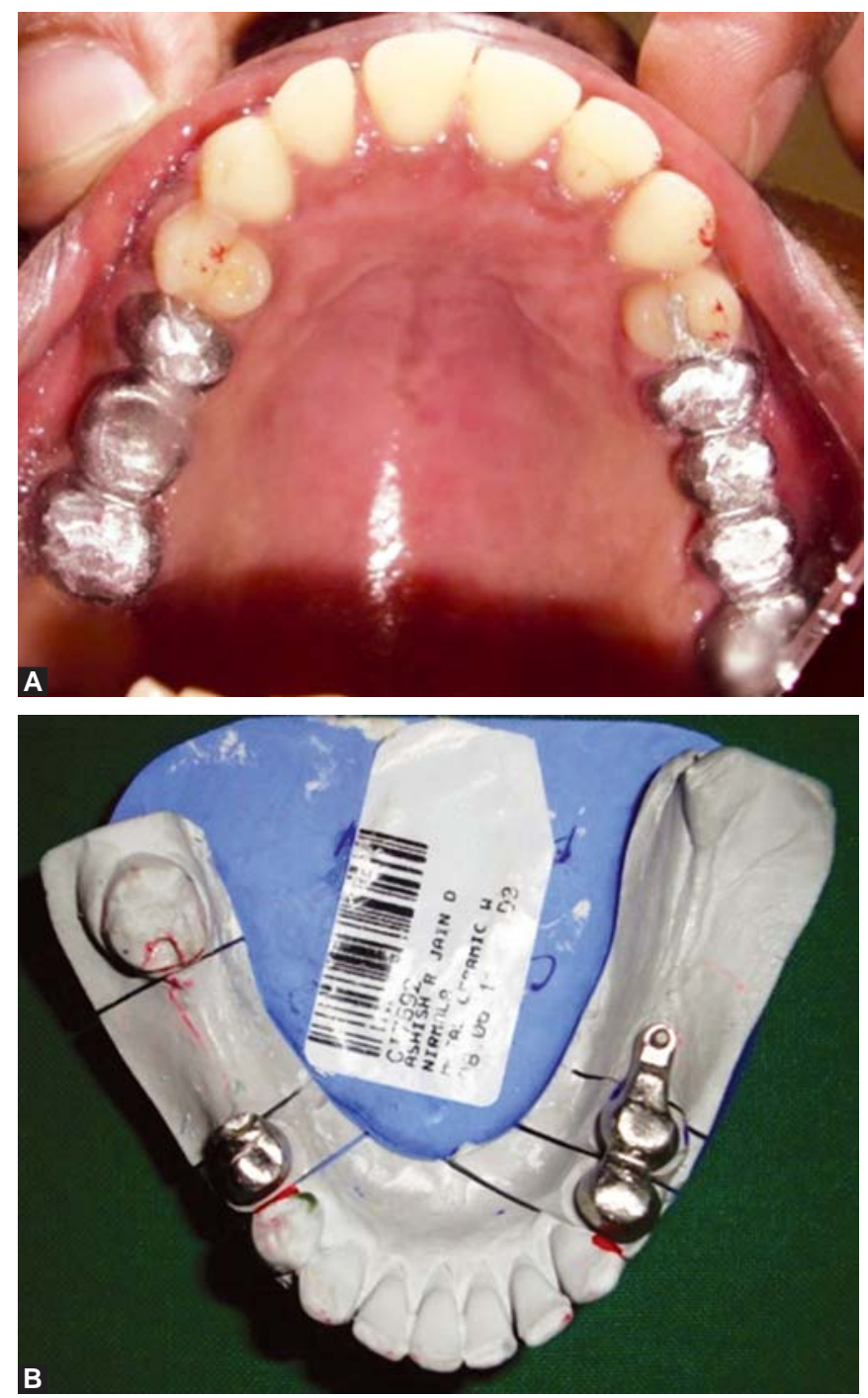

Figs 2A and B: Metal trial of the prosthesis
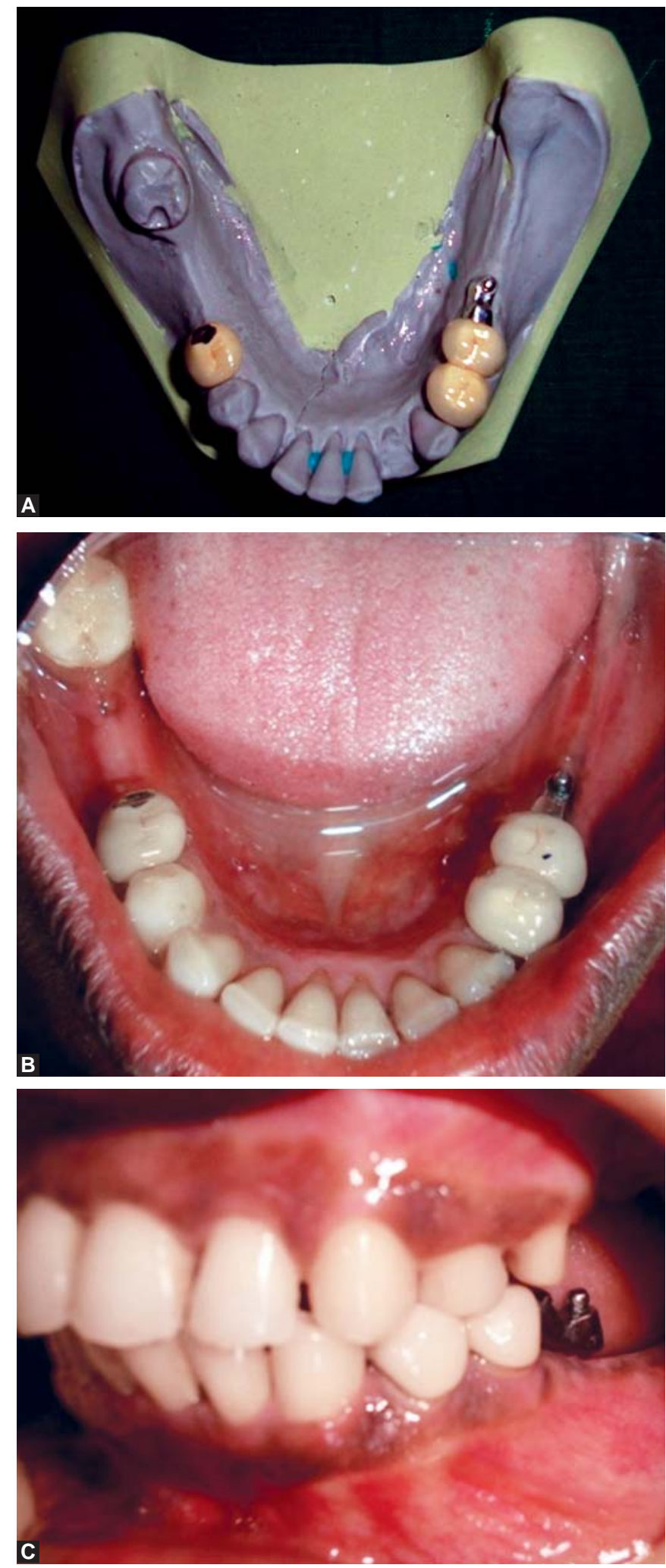

Figs 3A to C: P rovisional cementation of metal ceramic crowns in mandibular arch

- Cast was made from the impression and the regular fabrication of removal denture was done. Once the framework was fabricated, it was tried on the patient; maxillomandibular relationship was recorded, teeth arrangement and try-in was done and acrylization of RPD was performed (Figs 5A to C). 


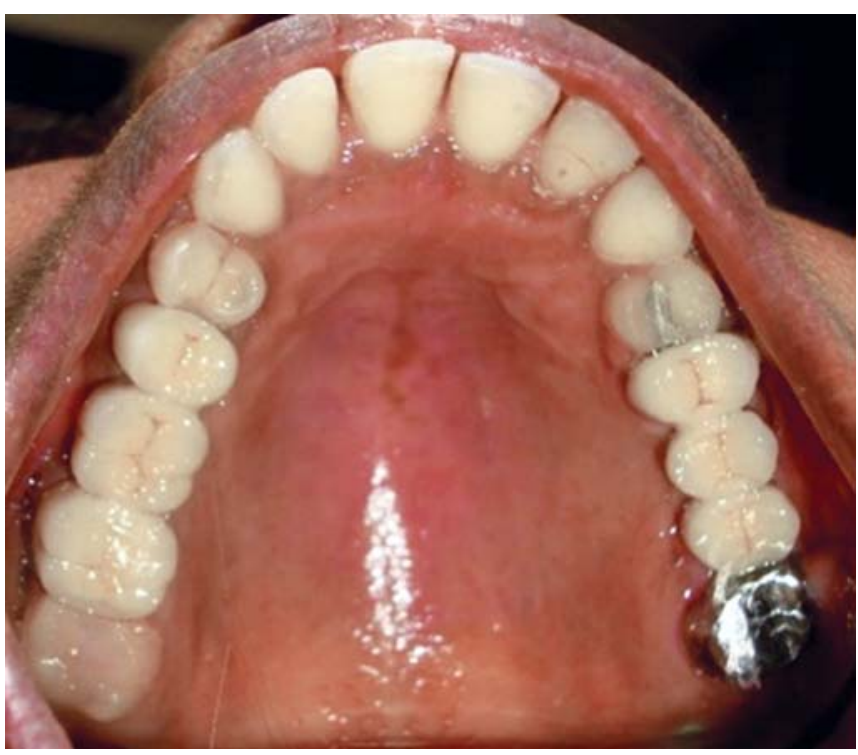

Fig. 4: Permanent cementation of metal ceramic crowns in maxillary arch

- M andibular FPD was cemented with glass ionomer cement. A fter the cement was set, the RPD was separated and excess cement from all areas was removed and occlusion verified (Figs $6 \mathrm{~A}$ and $\mathrm{B}$ ).

\section{DISCUSSION}

The disadvantages reportedly associated with RPD, such as patient discomfort, ill-fitting, loose prosthesis, decreased phonetics and masticatory efficiency were avoided. Cases of lower unilateral free end defects are the source of many problems in clinical dentistry. The denture design method most commonly used is cross-arch stabilization which involves creating a retainer on the side opposite the defect in order to stabilize the denture and protect the retaining teeth. Cross-arch bridges are used to stabilize teeth for patients with reduced periodontal support. Little is known about technical or biological complications of such treatment options. Whether fixed and removable prostheses can be combined for stability and the long-term effects of such treatment on tooth loss is yet to be seen. Cross-arch stabilizing bridges constructed for periodontal patients as part of their periodontal maintenance therapy had few complications and were associated with low rates of abutment tooth loss. Combining teeth and implants did not affect the performance of these bridges. ${ }^{7-9}$

Preiskel ${ }^{10}$ first reported the invention of attachment in early 20th century. To the late 20 century, with growing technology, the attachment has been applied to the superstructure of implant. Precision attachment has exceptional feature of being a removable prosthesis with improved esthetics, less postoperative adjustments and better patient comfort. ${ }^{7}$ The recommended procedure has
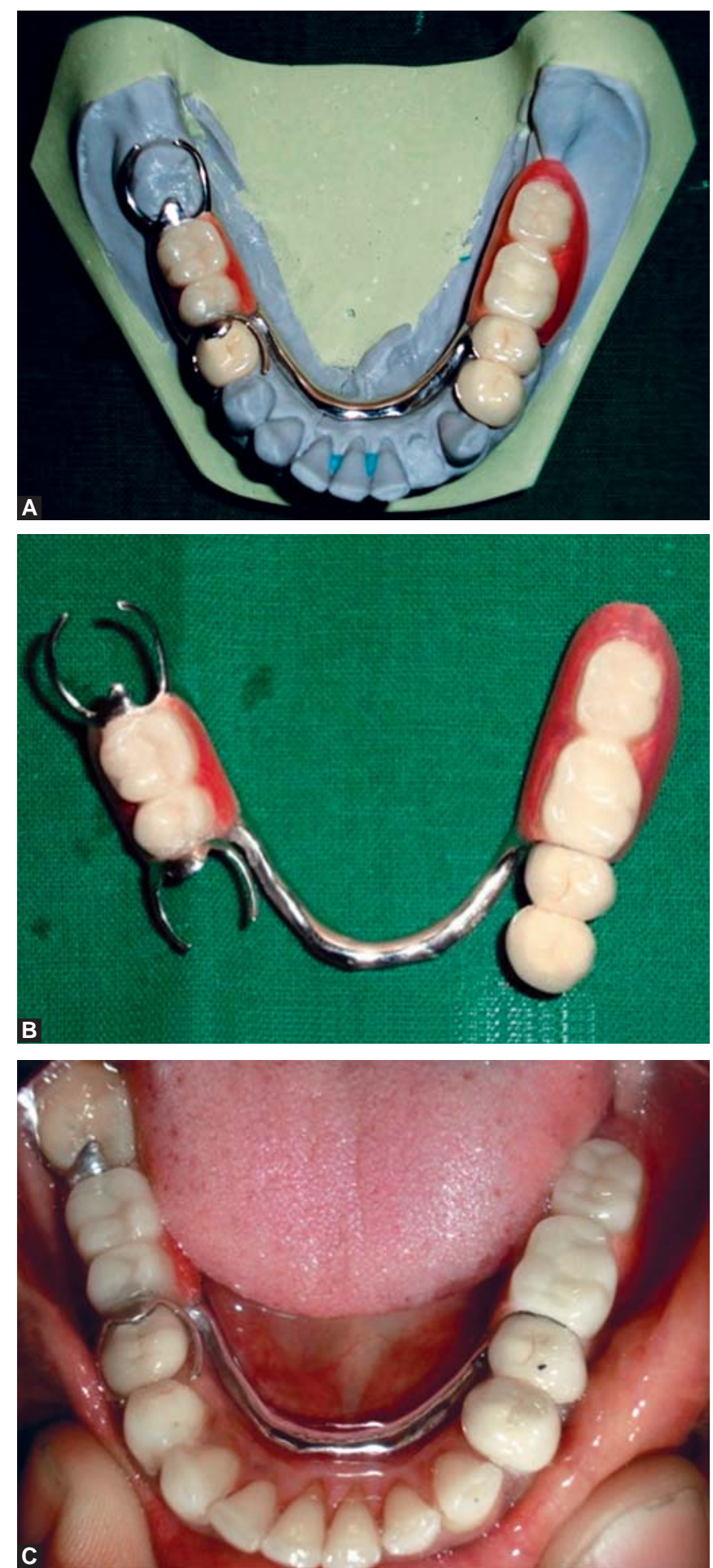

Figs $\mathbf{5 A}$ to $\mathbf{C}$ : Attachment retained and cast partial denture trial intraorally

several advantages over the conventional prosthesis. The advantages of attachment denture are beneficial and the limitation of RPD is reduced. Patient comfort and psychology are drastically improved. Being attachment prosthesis, the RPD can be removed and maintained as a regular denture. A dded to this, the laboratory procedures are simple, and treatment is economical compared to the more complex treatment options. ${ }^{11,12}$ The stress-control on 

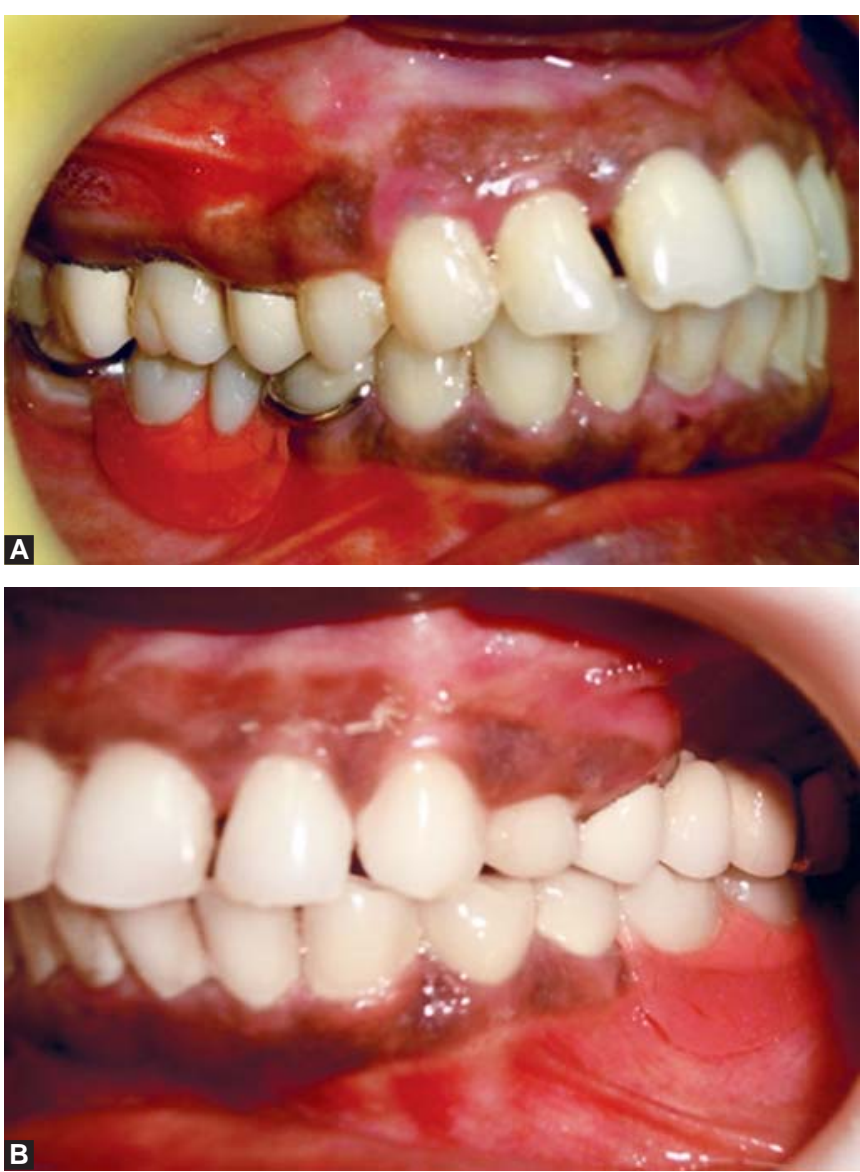

Figs 6A and B: Occlusion in right and left lateral view

abutment is an essential factor for the success of distal extension cast partial denture which is achieved through dual impression technique, broad coverage and stable denture base, rigid design, physiologic shimming, splinting of abutments, proper selection of attachment and clasp design. ${ }^{13,14}$ In this case report, abutments were of adequate clinical crown height to receive attachment; the extracoronal precision attachments for RPDs are recommended to this clinical situation because all teeth were involved as primary and secondary abutments for RPD tooth preparation. Extracoronal semiprecision attachments are easier to insert and remove. It is also esthetic and can be used for patients with limited manual dexterity, or when the prosthesis has a difficult path of insertion and removal. Extracoronal precision attachments are normally resilient and allow free movement of the prosthesis to distribute potentially destructive forces or loads away from the abutments to supportive bone and tissue. . $^{15,16}$

The use of a precision attachment-retained partial denture along with a cast metal framework has a biomechanical advantage over a unilateral precision attachment-retained partial denture without a cast metal framework in that it provides for cross-arch stabilization.
Though limitations exist with the attachment system, this Kennedy class 2 modification 1 partially edentulous situation attached with fixed or removable prosthesis has greater advantage clinically as discussed earlier.

\section{CONCLUSION}

This case report illustrates the option of using a removable prosthesis attached to a fixed prosthesis as a viable treatment option. The support of the RPD and its connection with the fixed prosthesis generates cross-arch stability throughout masticatory activity and permits function similar to that of a fixed denture. The use of the stress-director system effectively reduces metallic display and improves esthetics.

\section{REFERENCES}

1. Burns DR, Ward JE. A review of attachments for removable partial denture design: Part 1. Classification and selection. Int J Prosthodont 1990;3:98-102.

2. Burns DR, Ward JE. A review of attachments for removable partial denture design: Part 2. T reatment planning and attachment selection. Int J Prosthodont 1990;3:169-74.

3. Preiskel HW. Precision attachment in prosthodontics. $1 \& 2$. L ondon: Quintessence Publishing Co Ltd 1995:69-78.

4. Baker J L, Goodkind RJ. Precision attachment removable partial dentures. San M ateo, CA : M osby 1981:113-54.

5. Schuyler $\mathrm{CH}$. An analysis of the use and relative value of the precision attachment and the clasp in partial denture planning. J Prosthet Dent 1953;3:711-14.

6. Staubli PE. Attachments and implants: Reference manual (6th ed). San M ateo, CA : A ttachments International 1996:234-45.

7. F einberg $E$. Diagnosing and prescribing therapeutic attachmentretained partial dentures. NY S D ent J 1982;48(1):27-29.

8. Gillings BR. Magnetic retention for complete and partial dentures Part 1. J Prosthet D ent 1981;45:484.

9. Lorencki FS. Planning precision attachment restorations. J Prosthet Dent 1969;21(5):506-08.

10. Preiskel HW. Precision attachments in prosthodontics: Overdentures and telescopic prostheses. Chicago, IL, USA: Quintessence Publishing Co L td 1985;2.

11. Preiskel $H$. Precision attachments for free-end saddle prostheses. BrDent J 1969;127:462-68.

12. Picton $D C$, W illis DJ . V iscoelastic properties of the periodontal membrane and mucous membrane. J Prosthet Dent 1978;40: 263-72.

13. Kapur KK, Deupree R, Dent RJ, Hasse AL. A randomized clinical trail of two basic removable partial denture designs. Part I: Comparsion of five-year success rates and periodontal health. J Prosthet Dent 1994;72(3):268-82.

14. Kuzmanovic DV, Payne AG, Purton DG. Distal implants to modify the Kennedy classification of a removable partial denture: A clinical report. J Prosthet Dent 2004;92(1):8-11.

15. A viv I, B en-Ur Z, Cardash HS, Fatael H. RLS- the lingually retained clasp assembly for distal extension removable partial dentures. Quintessence Int 1990;21(3):221-23.

16. Owall B. Precision attachment-retained removable partial dentures: Part 2. Long-term study of ball attachments. Int J Prosthodont 1995;8(1):21-28. 


\section{ABOUT THE AUTHORS}

\section{Ashish R Jain (Corresponding Author)}

Senior L ecturer, Department of Prosthodontics, Tagore Dental College and Hospital, Chennai, Tamil Nadu, India

CorrespondenceAddress $\mathrm{R}$ House C $1, \mathrm{~N}$ o. 3, M anonmani A mmal Road, Pavapuri, Kilpauk, Chennai-600010, T amil Nadu, India, Phone: 09884233423, e-mail: dr.ashishjain_r@yahoo.com

\section{Jacob Mathew Philip}

Senior L ecturer, Department of Prosthodontics, Tagore Dental College and Hospital, Chennai, Tamil Nadu, India

\section{Padma Ariga}

Professor and Head, Department of Prosthodontics, Saveetha University, Chennai, Tamil Nadu, India 